\title{
At the dawn of personalized reproductive medicine: opportunities and challenges with incorporating multigene panel testing into fertility care
}

\author{
Piraye Yurttas Beim ${ }^{1}$ • David-Emlyn Parfitt ${ }^{1} \cdot$ Lei $_{\operatorname{Tan}^{1}}{ }^{1}$ Elaine A. Sugarman ${ }^{1}$. \\ Tina Hu-Seliger ${ }^{1} \cdot$ Caterina Clementi $^{1} \cdot$ Brynn Levy $^{2}$
}

Received: 10 June 2017 / Accepted: 13 October 2017 /Published online: 23 October 2017

(C) Springer Science+Business Media, LLC 2017

Advances in genomics are revolutionizing how diseases are prevented, diagnosed, and treated, across all fields of medicine. In reproductive medicine, genomic technologies are used for carrier, embryo, and prenatal screening. However, to date, genetic information has not been widely utilized for guiding treatment decisions or identifying individuals at risk for reproductive conditions, including those that can lead to infertility and early menopause. In contrast, analysis of genetic biomarkers is now the standard of care in oncology [1]. Today, oncologists routinely categorize tumors based on genetic signatures and refine their treatment strategies accordingly. This targeted approach to more personalized treatment has led to significantly higher progression-free survival rates when compared to the outcomes of conventional therapies [2]. Knowledge of patients' genetic risk factors has also decreased patient morbidity and mortality by more precisely revealing individuals who would benefit from preventative cancer screenings such as colonoscopies or mammograms.

The number of peer-reviewed articles investigating the genetics of reproductive conditions continues to grow (Fig. 1). Among these published studies is evidence suggesting that subclinical factors, for example dysregulation of ovarian follicle development, hormone metabolism, and immune system function, influence a woman's chances of experiencing reproductive difficulties [3-6]. Translation of these new findings into genetic testing panels that simultaneously interrogate multiple genetic biomarkers of risk for reproductive conditions is

Piraye Yurttas Beim

p.beim@celmatix.com

1 Celmatix Inc., New York, NY, USA

2 Department of Pathology \& Cell Biology, College of Physicians and Surgeons, Columbia University, New York, NY, USA now economically feasible given the decreasing cost of genetic sequencing. JARG recently published a review by Dr. Stephen Collins heralding this new era of personalized reproductive medicine [7]. In this article, Dr. Collins provides a clinician's perspective on both the opportunities and challenges of multigene panel testing, drawing on the lessons learned from the increasing use of genetic biomarkers in oncology.

The article highlights how multigene panel tests offer a superior time- and cost-effective option to assess genetic factors related to reproductive disorders by providing a simultaneous evaluation of multiple risk alleles. This observation is of particular importance, given the large out-of-pocket healthcare expenses faced by individuals seeking treatment for infertility or fertility preservation. The low cost-effectiveness of single gene tests has potentially contributed to the low adoption of genetic testing in reproductive medicine despite the growing menu of genetic tests that interrogate single factors related to female and male factor infertility. Multigene panels overcome this challenge by interrogating several relevant markers in a single test.

One opportunity not articulated in the Collins review is that multigene panel tests can potentially provide molecular, subclinical insights into a patient's unique reproductive physiology and function/dysfunction. Such insights are the true promise of personalized reproductive medicine and could help refine descriptive diagnoses such as recurrent pregnancy loss (RPL) or diminished ovarian reserve (DOR) to include underlying biological alterations disrupting key reproductive processes such as ovarian follicle development or embryo implantation. For example, current guidelines for clinical assessment of a patient experiencing RPL suggest evaluation of genetic factors such as parental karyotype, markers of thrombophilia when the patient has a personal or family history of venous thromboembolism, antiphosopholipid (AP) syndrome, uterine anatomy, and hormone, metabolic, and lifestyle 


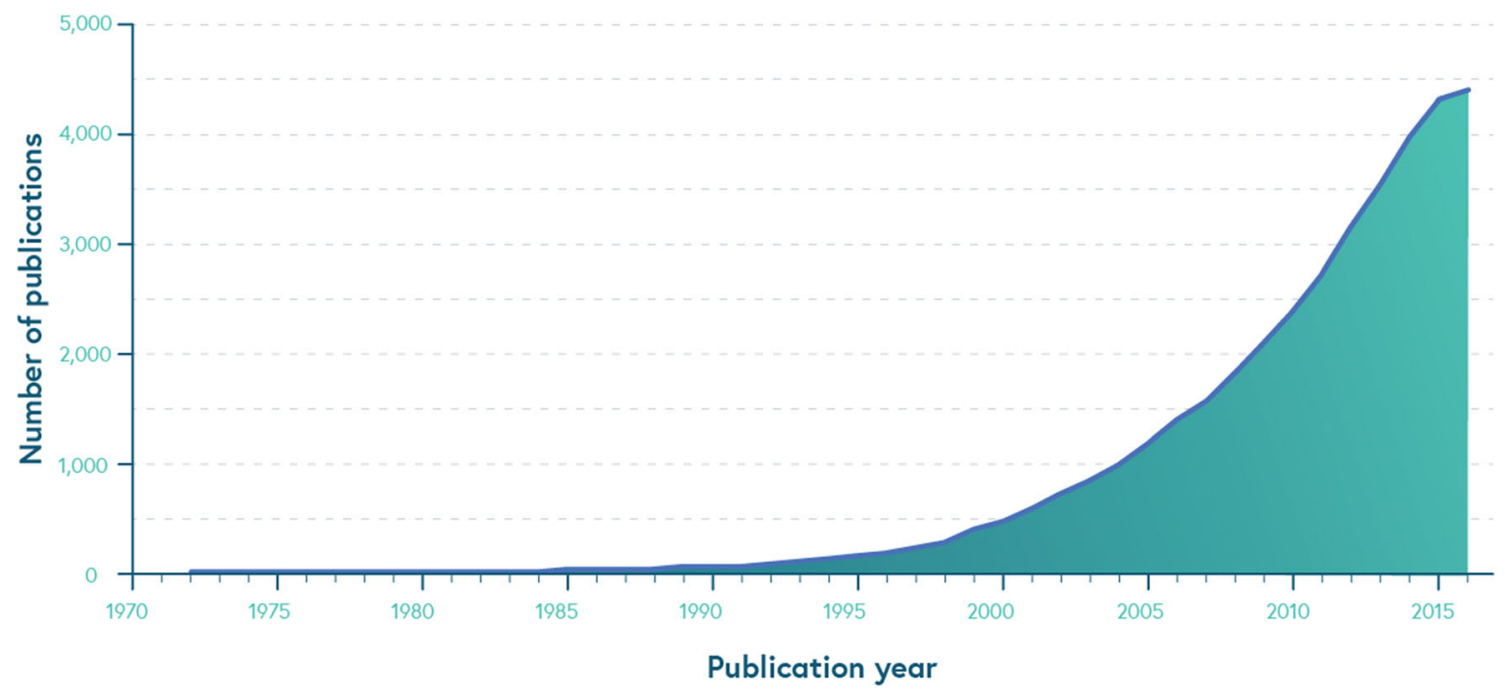

Fig. 1 Cumulative number of published genetic studies related to reproductive conditions, plotted by year. Types of studies include casecontrol association studies, linkage analysis studies, GWASs, and individual case reports, among others. Reproductive conditions include endometriosis, polycystic ovary syndrome, hyperandrogenism, recurrent

factors. The results of a standard assessment can lead to changes in treatment approaches. For example, patients in which AP antibodies are detected can be treated with a combination of unfractionated heparin and low-dose aspirin [8]. However, even after full RPL assessments, the root cause of up to $50 \%$ of RPL cases remains unexplained, meaning customized treatment options are thus limited. Multigene panel tests that reveal subclinical alterations in, for example, immune function or hormone metabolism, have the potential to suggest additional RPL risk factors that could lead to interventions based on a more refined understanding of the underlying etiology of the condition, in the same way that AP antibody detection does for a subset of RPL patients. The benefits of this strategy have already been realized in the oncology field, where particular drugs that initially failed clinical studies were later demonstrated to be effective after genetic stratification of patients or tumors [9]. Moreover, even when a clear intervention is not currently available, patients with unexplained RPL or infertility desire additional insight into genetic risk factors that could be contributing to their condition. In a US national survey of public perceptions of miscarriage, among study participants reporting a history of miscarriage, $47 \%$ reported feeling guilty, $41 \%$ reported feeling that they had done something wrong, and 38\% reported feeling they could have prevented it. Among all survey respondents, $78 \%$ indicated they would like to know the cause of miscarriage even if it would not help prevent future miscarriage [10]. Not being given any explanation for the cause of reproductive difficulties adds to the emotional, physical, and financial burden of infertility treatment for these individuals. Multigene panel tests, together with genetic counseling, hold the potential to improve both outcomes and the patient experience. pregnancy loss, recurrent implantation failure, diminished ovarian reserve, primary ovarian insufficiency, early menopause, idiopathic infertility, ovarian hyperstimulation syndrome, and response/outcome predictors

As with the introduction of any new technology, care must be taken to ensure that technological advances are leveraged appropriately to provide meaningful benefits to patients. Collins highlights some of the challenges related to bringing multigene panel tests to a new field of medicine, which are not unique to reproductive medicine. Prior to embracing a new genetic test, health care providers and the public expect evidence-based recommendations for its use. While multiple resources exist for establishing the diagnostic relationship between a gene/variant and Mendelian disorders [8], no standard framework currently exists for systematically assessing and statistically validating genetic risk factors associated with complex heterogeneous diseases like reproductive conditions. In the absence of established guidelines, it would be prudent to use the conceptual principles of a resource such as the Clinical Genome (ClinGen) Gene-Disease Clinical Validity Classification Framework [11] to rank and evaluate gene- and variant-level associations with reproductive conditions. One could argue that only variants that fall within genetic loci with strong evidence linking them to a reproductive condition and that hold up to rigorous statistical validation analysis should be incorporated into multigene testing panels. Also, since multigene panel tests are not regulated by the FDA, but rather through individual state departments of health, it is important for physicians to understand the regulatory standards for genetic testing in each state and recognize that these standards are not uniform throughout the country.

Reporting genetic test results from complex disorders is likely to be fundamentally different from traditional genetic tests for monogenic disorders, especially since results may be 
more appropriately presented as risk factors as opposed to diagnostic markers. Genetic testing for risk factors, which by definition is probabilistic and not diagnostic, will always have inherent challenges. One challenge raised by Collins is where to draw the line on extent of risk, often interpreted and/or estimated from odds ratios (ORs). To exemplify this, Collins focuses on the subset of genetic markers of the Fertilome ${ }^{\circledR}$ test related to primary ovarian insufficiency (POI), which has ORs ranging from 1.5 to 4.5. Collins suggests that the bar for clinical utility should be higher, due to low absolute risk. However, risk factors with comparable odds ratios have made significant clinical and societal impacts, even in the context of low absolute risk. For example, prone sleeping position is associated with an increased risk of sudden infant death syndrome (SIDS) when compared to back sleeping position, with a pooled odds ratio of 4.46 (from a meta-analysis of 25 studies) [12]. Though the absolute risk of SIDS was only around one in every 1000 births at the time [13], the identification of prone sleeping as a risk factor still influenced the launch of a national "Back to Sleep" campaign in 1994 by the National Institute of Child Health and Development [14]. In the 10 years after initiating this campaign, the SIDS rate declined by $>50 \%$ [15], which is considered to be a spectacular achievement for public health [16]. Smoking exposure is another relevant example. A meta-analysis of 12 studies revealed an overall OR of 1.60 for risk of infertility in women smokers vs. non-smokers [17]. Despite the relatively low OR, the American Society for Reproductive Medicine and the Society for Reproductive Endocrinology and Infertility both consider smoking to negatively impact fertility and consequently recommend avoidance for couples trying to conceive [18]. Thus, low absolute risk should not, in of itself, be a sole determinant of whether genetic risk factors should be incorporated into patient counseling. As with SIDS intervention or smoking cessation, there are clear actions that can be taken if a woman is identified as carrying a genetic risk factor for POI. These can range from closer monitoring of her hormone levels through her reproductive years, to fertility preservation through egg freezing. Genetic information may also influence some women to start families earlier in their lives or avoid environmental and lifestyle risk factors that may further accelerate decline of their ovarian reserve. Everyone has a different level of risk tolerance, and drawing an absolute line at an arbitrary odds ratio for a particular genetic variant may not be in the best interest of all patients. Some women may choose to make changes based on relatively low levels of risk. Further, absolute risk is less relevant than the accurate assessment of risk based on statistically significant odds ratios calculated from data reported by wellcontrolled studies. Choosing variants that meet these criteria allows clinicians and patients to assess risk in the context of a woman's personal clinical situation and preferences. Without giving women access to this genetic risk information, this choice is effectively taken away.
One additional point raised in the Collins review that warrants discussion concerns the term "pathogenic" when applied to a genetic variant. "Pathogenic" is used to denote a gene alteration that causes disease in a particular context [19]. In the context of infertility, a complex and heterogeneous condition, it is important to recognize that a variant's demonstrated impact on protein function may not be sufficient to result in a given phenotype. Therefore, gene alterations functionally implicated in biological processes related to a gene may not be appropriate candidates for an infertility gene panel in the absence of multiple independent studies demonstrating a clinical association in affected individuals. As an example, the $Z P 1$ gene is part of the "infertility" or "POI" panel for six different commercial entities. The ZP1 protein, one of the glycoproteins that make up the zona pellucida, has been well studied from a functional perspective in vitro and in mouse models [20,21]. However, only one paper has linked alterations in this gene to possible reproductive difficulties in humans [22]. The paper, by Huang et al., describes a homozygous frameshift mutation in $Z P 1$ in six family members. The authors themselves make the argument that consanguinity significantly weakens the results of this study. This underscores the necessity of physicians to familiarize themselves with the science behind gene panels and to hold the laboratories conducting these assays accountable for doing due diligence in assessing the strength of the evidence linking the variants included on the commercial gene panels to the various reproductive conditions and ensure that the associations hold up to rigorous statistical standards.

Another challenge raised by the Collins review is in the generalizability of associations in various racial and ethnic populations to which they are being applied. For example, the rs254286 variant, within the GDF9 gene, has been assessed for its association with POI in both Indian [23] and Chinese [24] cohorts. Collins expressed concerns that this variant has only been studied in these "Asian" populations and therefore may not be broadly applicable. It should be noted that while these study populations are geographically "Asian," South Asians (SAS) are genetically distinct from East Asians (EAS) [25], and the allele frequencies for this variant from these two super-populations vary substantially ( 0.29 for EAS and 0.62 for SAS). We certainly agree that it is important to verify and track the association of a new marker in different populations. However, for the GDF9 variant, an association with POI was independently validated in a metaanalysis of two distinct populations with very different allele frequencies, providing compelling evidence in support of the association.

It seems likely that in the future, we will look back in amazement and wonder at how reproductive medicine was practiced without the personalization and molecular insights provided by genetic testing. In the years to come, we can expect different sub-specialties to emerge, with physicians speaking a newly-defined language that is less grounded in 
hormone levels and ultrasound measurements and based more on individual genetic profiles that relate to specific biological processes. It is exciting to be at the dawn of this new era, but there remains a burden of responsibility on those developing and implementing these groundbreaking technologies to show concrete evidence of clinical utility and patient benefits. Drawing on the example of targeted cancer therapeutics as a guide, it is anticipated that the provision of more personalized explanations for reproductive conditions will also be accompanied by a growing menu of molecularly targeted interventions with fewer side effects and higher success rates. Ultimately, the era of personalized reproductive medicine will enable a shift from current reactive treatments for reproductive issues to the proactive management of lifelong reproductive potential.

\section{Compliance with ethical standards}

Conflict of interest PYB, DEP, LT, EAS, THS, and CC are employees of Celmatix Inc. BL is a consultant for Celmatix Inc.

\section{References}

1. ACOG Practice Bulletin No. 103: Hereditary breast and ovarian cancer syndrome. Obstet Gynecol. 2009;113(4):957-66. https:// doi.org/10.1097/AOG.0b013e3181a106d4.

2. Massard C, Michiels S, Ferte C, Le Deley MC, Lacroix L, Hollebecque A, et al. High-throughput genomics and clinical outcome in hard-to-treat advanced cancers: results of the MOSCATO 01 trial. Cancer Discov. 2017;7(6):586-95. https://doi.org/10.1158/ 2159-8290.CD-16-1396.

3. Hannan NJ, Salamonsen LA. Role of chemokines in the endometrium and in embryo implantation. Curr Opin Obstet Gynecol. 2007;19(3): 266-72. https://doi.org/10.1097/GCO.0b013e328133885f.

4. Boots CE, Jungheim ES. Inflammation and human ovarian follicular dynamics. Semin Reprod Med. 2015;33(4):270-5. https://doi. org/10.1055/s-0035-1554928.

5. Henriet P, Gaide Chevronnay HP, Marbaix E. The endocrine and paracrine control of menstruation. Mol Cell Endocrinol. 2012;358(2):197-207. https://doi.org/10.1016/j.mce.2011.07.042.

6. Makker A, Goel MM, Mahdi AA. PI3K/PTEN/Akt and TSC/ mTOR signaling pathways, ovarian dysfunction, and infertility: an update. J Mol Endocrinol. 2014;53(3):R103-18. https://doi. org/10.1530/JME-14-0220.

7. Collins SC. Precision reproductive medicine: multigene panel testing for infertility risk assessment. J Assist Reprod Genet. 2017;34(8):967-73. https://doi.org/10.1007/s10815-017-0938-y.

8. Practice Committee of the American Society for Reproductive M. Evaluation and treatment of recurrent pregnancy loss: a committee opinion. Fertil Steril. 2012;98(5):1103-11. https://doi.org/10.1016/ j.fertnstert.2012.06.048.

9. Keedy VL, Temin S, Somerfield MR, Beasley MB, Johnson DH, McShane LM, et al. American Society of Clinical Oncology provisional clinical opinion: epidermal growth factor receptor (EGFR) mutation testing for patients with advanced non-small-cell lung cancer considering first-line EGFR tyrosine kinase inhibitor therapy. J Clin Oncol. 2011;29(15):2121-7. https://doi.org/10. 1200/JCO.2010.31.8923.

10. Bardos J, Hercz D, Friedenthal J, Missmer SA, Williams Z. A national survey on public perceptions of miscarriage. Obstet Gynecol. 2015;125(6):1313-20. https://doi.org/10.1097/AOG. 0000000000000859.

11. Strande NT, Riggs ER, Buchanan AH, Ceyhan-Birsoy O, DiStefano M, Dwight SS, et al. Evaluating the clinical validity of gene-disease associations: an evidence-based framework developed by the clinical genome resource. Am J Hum Genet. 2017;100(6):895-906. https://doi.org/10.1016/j.ajhg.2017.04.015.

12. Gilbert R, Salanti G, Harden M, See S. Infant sleeping position and the sudden infant death syndrome: systematic review of observational studies and historical review of recommendations from 1940 to 2002. Int J Epidemiol. 2005;34(4):874-87. https://doi.org/10. 1093/ije/dyi088.

13. CDC | Sudden unexpected infant death and sudden infant death syndrome. https://www.cdc.gov/sids/data.htm. 2017.

14. Willinger M, Hoffman HJ, Hartford RB. Infant sleep position and risk for sudden infant death syndrome: report of meeting held January 13 and 14, 1994, National Institutes of Health, Bethesda, MD. Pediatrics. 1994;93(5):814-9.

15. American Academy of Pediatrics Task Force on Sudden Infant Death S. The changing concept of sudden infant death syndrome: diagnostic coding shifts, controversies regarding the sleeping environment, and new variables to consider in reducing risk. Pediatrics. 2005;116(5):1245-55. https://doi. org/10.1542/peds.2005-1499.

16. Trachtenberg FL, Haas EA, Kinney HC, Stanley C, Krous HF. Risk factor changes for sudden infant death syndrome after initiation of back-to-sleep campaign. Pediatrics. 2012;129(4):630-8. https://doi. org/10.1542/peds.2011-1419.

17. Augood C, Duckitt K, Templeton AA. Smoking and female infertility: a systematic review and meta-analysis. Hum Reprod. 1998;13(6):1532-9.

18. Practice Committee of the American Society for Reproductive Medicine in collaboration with the Society for Reproductive Endocrinology and Infertility. Optimizing natural fertility: a committee opinion. Fertil Steril 2017;107(1):52-58. https://doi.org/10. 1016/j.fertnstert.2016.09.029.

19. Human Genome Variation Society | Sequence Variant Nomenclature. http://varnomen.hgvs.org/bg-material/basics/. 2017.

20. Ganguly A, Bukovsky A, Sharma RK, Bansal P, Bhandari B, Gupta SK. In humans, zona pellucida glycoprotein-1 binds to spermatozoa and induces acrosomal exocytosis. Hum Reprod. 2010;25(7):1643-56. https://doi.org/10.1093/humrep/deq105.

21. Rankin T, Talbot P, Lee E, Dean J. Abnormal zonae pellucidae in mice lacking ZP1 result in early embryonic loss. Development. 1999;126(17):3847-55.

22. Huang HL, Lv C, Zhao YC, Li W, He XM, Li P, et al. Mutant ZP1 in familial infertility. N Engl J Med. 2014;370(13):1220-6. https:// doi.org/10.1056/NEJMoa1308851.

23. Dixit H, Rao LK, Padmalatha V, Kanakavalli M, Deenadayal M, Gupta N, et al. Mutational screening of the coding region of growth differentiation factor 9 gene in Indian women with ovarian failure. Menopause. 2005;12(6):749-54. https://doi.org/10.1097/01.gme. 0000184424.96437.7a.

24. Ma L, Chen Y, Mei S, Liu C, Ma X, Li Y, et al. Single nucleotide polymorphisms in premature ovarian failure-associated genes in a Chinese Hui population. Mol Med Rep. 2015;12(2):2529-38. https://doi.org/10.3892/mmr.2015.3762.

25. Genomes $\mid$ A deep catalog of human genetic variation. http://www. internationalgenome.org/home. 2017. 